\title{
Hybrid Modeling of Molten Steel Temperature Prediction in LF
}

\author{
Huixin TIAN, Zhizhong MAO and Yan WANG \\ Information Science and Engineering College, P.O. Box, No. 131, Northeastern University, Shenyang, 110004, P. R. China. \\ E-mail: icedewl@163.com
}

(Received on May 28, 2007; accepted on November 7, 2007)

\begin{abstract}
Combining the conventional mechanism method with the newly developed intelligent algorithm, a hybrid model was developed for predicting the molten steel temperature in LF. The mechanism method is used to build the thermal model by analyzing the energy going into and out of the molten steel during the LF refining production process. It is hard to calculate the coefficients in the thermal model by mechanism method, so they are estimated by experience traditionally. In this paper, a new ensemble ELM algorithm using the modified AdaBoost.RT is proposed to calculate these coefficients. The new hybrid model overcomes the difficulty of obtaining the coefficients in thermal model, and solves the problem of limited prediction precision using "black box" model. The experiment demonstrates that the new hybrid model can improve generalization performance and the prediction accuracy.
\end{abstract}

KEY WORDS: hybrid model; ladle furnace; ELM; thermal model; mechanism method; intelligent algorithm.

\section{Introduction}

At present, Ladle furnace (LF) is used extensively in the iron and steel industry. LF refining technology plays a substantial role in secondary metallurgic process. The main purpose of ladle furnace treatment is to ensure that the molten steel has the required temperature when the ladle is taken over at downstream secondary metallurgy units or at a continuous caster. Therefore, the temperature control of LF is essential for improving both productivity and product quality. The temperature foreseeing is the key factor for the temperature control of LF successfully.

Several LF models based on thermodynamics and conservation of energy were developed in earlier works. For example, a thermodynamic analysis that based on the second law of thermodynamics was presented for molten steel by U. Camdali et al. ${ }^{1)}$ Similarly, the physical exergy during the steel production process such as loss exergy, exergies going into and out of the LF were evaluated and the ways of minimizing these losses in practice are discussed by $\mathrm{U}$. Camdali and M. Tunc. ${ }^{2,3)}$ These models were extremely useful thermal analysis tool and were very accurate. However, these models cannot be used for on-line prediction as the parameters were hard to obtain. It is attributed to the harsh operating environment of ladle metallurgy especially the high temperatures and corrosive slag associated with the process.

To overcome these limitations, the "black box" models based on the data were presented. S. Yanguang et al. used artificial intelligent technology to predict the temperature of LF. ${ }^{4)}$ The shortage of these "black box" models is the limited prediction precision for lacking the analysis of ladle furnace metallurgy process.

In this paper, a new hybrid model combined the mecha- nism method with intelligent algorithm is proposed. The new model overcomes the difficulty of obtaining the coefficients in mechanism model and the limitation of lower prediction precision using the "black box" model.

\section{Thermal Model of Ladle Furnace}

For the simplified on-line model of the LF process, liquid steel is assumed to be homogeneous with respect to temperature and composition throughout the bath, and one-dimensional variation of temperature profile is assumed in the ladle refractory wall. A thermal model has been developed for $300 \mathrm{t}$ LF of Baoshan Iron \& Steel Co. Ltd. based on conservation of energy. Between any two measured temperatures there may be various operations taking place such as purging, arcing, additions and so on, so that the rise or fall in temperature would be due to the combined effect of all the operations. Loss exergy, exergies going into and out of the LF are identified and exergy efficiency of the system is determined. ${ }^{1-3,5)}$ The mathematical thermal model is classified into the following sections:

(i ) Heat gain due to arcing

(ii) Heat loss of the refractory wall

(iii) Heat effects of additions

(iv) Heat loss from the top surface

\subsection{Heat Gain Due to Arcing}

The heat LF gained is from arcing. The submerged arc operation ensures the heat gain of molten steel. The following expression is employed to describe the change in temperature due to arcing.

$$
\Delta T_{\mathrm{arc}}=\eta E
$$

Where, $\eta$ is the thermal efficiency coefficient of arcing. $E$ 
Table 1. Temperature drop coefficients when charging $300 t$ LF with metal alloy and slag.

\begin{tabular}{cc|cc}
\hline Addition & $\begin{array}{c}\text { temperature effects } \\
\text { parameter } \times 10^{-2} \\
\left({ }^{\circ} \mathrm{C} / \mathrm{kg}\right)\end{array}$ & Addition & $\begin{array}{c}\text { temperature effects } \\
\text { parameter } \times 10^{-2} \\
\left({ }^{\circ} \mathrm{C} / \mathrm{kg}\right)\end{array}$ \\
\hline $\mathrm{HcFeCr}$ & -0.95 & $\mathrm{LcFeCr}$ & -0.65 \\
$\mathrm{HcFeMn}$ & -0.9 & $\mathrm{LcFeMn}$ & -0.75 \\
$\mathrm{FeMo}$ & -0.75 & $\mathrm{FeSi}$ & +0.9 \\
$\mathrm{Slag}$ & -1.0 & $\mathrm{C}$ & -2.5 \\
$\mathrm{Al}$ & +5.0 & $\mathrm{Ni}$ & -0.5 \\
$\mathrm{FeNb}$ & -0.35 & $\mathrm{FeTi}$ & -0.4 \\
$\mathrm{CaSi}$ & -1.05 & $\mathrm{FeAl}$ & +1.0 \\
$\mathrm{HCZl}$ & -1.0 & $\mathrm{Al}-\mathrm{D}$ & -0.5 \\
$\mathrm{CaO}$ & -1.0 & $\mathrm{LCCR}$ & -0.65 \\
\hline
\end{tabular}

is the total refining power consumption.

\subsection{Heat Loss of the Ladle Refractory Wall}

Temperature drop due to heat loss during the heating of refractory can be seen as a one-dimensional transient heat transfer equation for the side wall and bottom wall. ${ }^{6}$ It is calculated with the following assumptions and expression.

Initial condition: $t=0, T_{\text {Wall }}=T_{\text {Preht }}$

Boundary condition: $t=t, T_{\text {Wall }}=T_{\text {LiqSt }}$

$$
\rho C_{\mathrm{P}} \frac{d T_{\text {Wall }}}{d t}=h\left(T_{\text {Wall }}-T_{\infty}\right)
$$

Where, $T_{\text {Preht }}$ is the temperature of preheated refractory. $T_{\text {LiqSt }}$ is temperature of liquid steel. $T_{\infty}$ is the temperature of the plant environment. $\rho$ is the density of steel $\left(\mathrm{kg} / \mathrm{m}^{3}\right) . C_{\mathrm{P}}$ is the specific heat of steel $\left(\mathrm{J} \mathrm{K}^{-1} \mathrm{~kg}\right) . h$ is the heat transfer coefficient $\left(\mathrm{J} / \mathrm{m}^{2} \mathrm{~s}\right)$. Then $\Delta T_{\text {Wall }}$ owing to the refractory heat loss can be calculated.

\subsection{Heat Effects of Additions}

The temperature change of liquid steel owing to additions is shown as the following expression:

$$
\Delta T_{\mathrm{add}}=\sum_{i} W_{i} Q_{i}
$$

Where $i$ designates a specific addition (metal alloy or slag). $W_{i}$ is the weight of addition $i(\mathrm{~kg}) . Q_{i}$ is the temperature effect parameter of $i\left({ }^{\circ} \mathrm{C} / \mathrm{kg}\right)$. The statistical average of the temperature effects of various additions is shown in Table 1.

\subsection{Heat Loss from the Top Surface}

The heat loss from the top surface is mainly due to the radiation loss of molten slag surface and furnace cover. Moreover, the radiation loss of molten slag surface is owing to the temperature of molten slag, quantification of slag and surface area. It is difficult to obtain the quantification of molten slag exactly. And the temperature of liquid steel ${ }^{7)}$ (replacing the slag temperature in mechanism model) is changing continually during the LF metallurgy process. Therefore, it is hard to calculate the heat loss from the top surface exactly using the traditional mechanism model. The heat loss is obtained by calculating the energy change of the cooling water in this paper.

$$
\Delta T_{\text {Surf }}=\frac{f_{\text {Surf }} C_{\mathrm{w}} Q_{\mathrm{w}} \Delta T_{\mathrm{w}}}{C_{\mathrm{p}} W}
$$

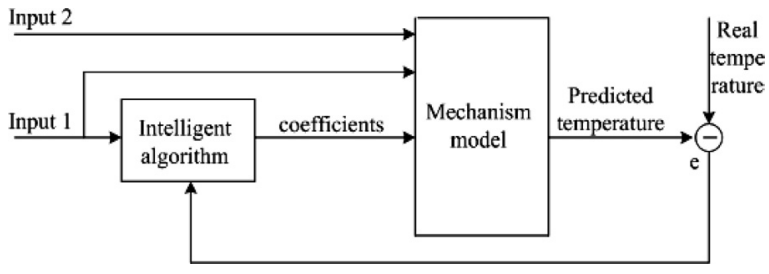

Fig. 1. Hybrid modeling of prediction of molten steel temperature.

Where, $f_{\text {Surf }}$ is the heat loss coefficient of slag surface and furnace cover. $C_{\mathrm{w}}$ is specific heat of cooling water $\left(\mathrm{J} \mathrm{K}^{-1} \mathrm{~kg}\right) \cdot Q_{\mathrm{w}}$ is the flux of cooling water $(\mathrm{L} / \mathrm{s}) . \Delta T_{\mathrm{w}}$ is the temperature change of cooling water. $W$ is the weight of liquid steel.

\subsection{Overall Thermal Model}

The complete thermal model for the prediction of temperature in LF can be described as the total effect of the above factors:

$$
\Delta T=\Delta T_{\text {arc }}+\Delta T_{\text {Wall }}+\Delta T_{\text {add }}+\Delta T_{\text {Surf }} \cdot
$$

\section{Hybrid Model}

The heat LF gained is mainly from the arc which is submerged in slag. Therefore, the value of $\eta$ depends on the quality of submerged arc. On the other hand, the heat loss from the electrode and short-supply network can not be ignored, so it is hard to calculate $\eta$ by mechanism method. At the same time, the coefficient $f_{\text {Surf }}$ is related to the flux of cooling water, the quantification of slag and so on. It faces the same problem as the coefficient $\eta$. In traditional mechanism model, these coefficients are all estimated by experience. In this paper, a hybrid modeling method combined the intelligent algorithm with the mechanism method is proposed. The coefficients are calculated by intelligent algorithm firstly. And then, the temperature of molten steel is calculated by mechanism model with these coefficients. The error of real temperature and predicted temperature is used to modify the hybrid model for better accuracy (Fig. 1). This new hybrid model can solve the problem that the coefficients are calculated hardly in mechanism model. It also overcomes the problems of "black box" such as bad generalization performance, lack of the analysis of ladle furnace metallurgy process and so on. The ensemble ELM algorithm is used in the hybrid model. This new intelligent algorithm can improve generalization performance and the prediction accuracy.

\section{Extreme Learning Machine}

A novel learning algorithm for single-hidden-layer feedforward neural networks called extreme learning machine (ELM) was proposed by Huang et al. recently. ${ }^{8)}$ In ELM, the input weights (linking the input layer to the hidden layer) and hidden biases are randomly chosen, and the output weights (linking the hidden layer to the output layer) are analytically determined by using Moore-Penrose (MP) generalized inverse. Unlike traditional neural networks, the ELM algorithm needs to calibrate the parameters (learning 
rate, learning epochs, etc.) on more. The learning speed of ELM is extremely fast compared to other traditional methods. The traditional classic gradient-based learning algorithms may face several issues like local minima, improper learning rate and overfitting, etc. In order to avoid these issues, some methods such as weight decay and early stopping methods need to be used often in these classical learning algorithms. The ELM tends to obtain the solutions straightforward without such trivial issues. The ELM learning algorithm looks much simpler than most learning algorithms for feedforward neural networks. So in this paper an ensemble ELM is proposed to predict the coefficients of mechanism model.

\subsection{Single Hidden Layer Feedforward Networks (SLFNs)}

For $N$ arbitrary distinct samples $\left\{\left(x_{j}, t_{j}\right)\right\}_{j=1}^{N}$, where $x_{j}=$ $\left[x_{j 1}, x_{j 2}, \cdots, x_{j n}\right]_{\tilde{N}}^{T} \in R^{n}$ and $t_{j}=\left[t_{j 1}, t_{j 2}, \cdots, t_{j m}\right]^{T} \in R^{n}$, a standard SLFNs with $\tilde{N}$ hidden nodes and activation function $g(x)$ are mathematically modeled as

$$
\sum_{i=1}^{\tilde{N}} \beta_{i} g_{i}\left(x_{j}\right)=\sum_{i=1}^{\tilde{N}} \beta_{i} g\left(w_{i} \cdot x_{j}+b_{i}\right)=o_{j}, \quad j=1, \cdots, N
$$

where $w_{i}=\left[w_{i 1}, w_{i 2}, \cdots, w_{i n}\right]^{T}$ is the weight vector connecting the $i$ th hidden node and the input nodes, $\beta_{i}=\left[\beta_{i 1}, \beta_{i 2}, \cdots\right.$, $\left.\beta_{i m}\right]^{T}$ is the weight vector connecting the $i$ th hidden node and the output nodes, $o_{j}=\left[o_{j 1}, o_{j 2}, \cdots, o_{j m}\right]^{T}$ is the output vector of the SLFN, and $b_{i}$ is the threshold of the $i$ th hidden node. $w_{i} \cdot x_{j}$ denotes the inner product of $w_{i}$ and $x_{j}$. The output nodes are chosen linear. The standard SLFNs with $\tilde{N}$ hidden nodes and activation function $g(x)$ can approximate these $N$ samples with zero error means that $\sum_{j=1}^{\tilde{N}}\left\|o_{j}-t_{j}\right\|=0$. And these $N$ equations can be written compactly as:

$$
\mathrm{H} \beta=\mathrm{T}
$$

where

$$
\begin{array}{r}
\mathrm{H}=\left[\begin{array}{ccc}
g\left(w_{1} \cdot x_{1}+b_{1}\right) & \cdots & g\left(w_{\tilde{N}} \cdot x_{1}+b_{\tilde{N}}\right) \\
\vdots & \cdots & \vdots \\
g\left(w_{1} \cdot x_{N}+b_{1}\right) & \cdots & g\left(w_{\tilde{N}} \cdot x_{N}+b_{\tilde{N}}\right)
\end{array}\right]_{N \times \tilde{N}} \\
\beta=\left[\begin{array}{c}
\beta_{1}^{T} \\
\vdots \\
\beta_{\tilde{N}}^{T}
\end{array}\right]_{\tilde{N} \times m}
\end{array}
$$

and

$$
\mathrm{T}=\left[\begin{array}{c}
t_{1}^{T} \\
\vdots \\
t_{N}^{T}
\end{array}\right]_{N \times m}
$$

Where $\mathrm{H}$ is called the hidden layer output matrix.

\subsection{ELM Algorithm}

In most case, the number of hidden nodes is much less than the number of training samples $(\tilde{N} \ll N)$. It means that $\mathrm{H}$ is a nonsquare matrix and there may not exist $w_{i}, b_{i}, \beta_{i}$ $(i=1, \cdots, \tilde{N})$ such that $\mathrm{H} \beta=\mathrm{T}$. The parameters of hidden nodes need not be tuned and can be randomly generated permanently according to any continuous probability distribution. The unique smallest norm least squares solution of the above linear system is

$$
\hat{\beta}=\mathrm{H}^{+} \mathrm{T} \text {. }
$$

Where $\mathrm{H}^{+}$is the Moore-Penrose generalized inverse of matrix $\mathrm{H}$.

Thus, a simple learning method for SLFNs called extreme learning machine (ELM) can be summarized as follows:

Step 1: Randomly assign input weight $w_{i}$ and bias $b_{i}$, $i=1, \cdots, \tilde{N}$

Step 2: Calculate the hidden layer output matrix $\mathrm{H}$

Step 3: Calculate the output weight $\beta: \beta=\mathrm{H}^{+} \mathrm{T}$

The universal approximation capability of ELM has been rigorously proved in an incremental method by Huang et al. ${ }^{8)}$

\section{Ensemble ELM}

In this paper a modified AdaBoost.RT algorithm for regression is proposed to improve the performance of single ELM as an ensembles method. Ensembles methods can improve the generalization performance and the prediction accuracy. ELM is used as "weak learner" of the modified AdaBoost.RT.

\subsection{Adaboost.RT Algorithm}

A new boosting algorithm for regression problems, AdaBoost.RT ( $\mathrm{R}$ stands for regression and T for threshold) ${ }^{9,10)}$ is described as follows:

1) Input:

Sequence of $m$ examples $\left(x_{1}, y_{1}\right), \cdots,\left(x_{m}, y_{m}\right)$, where output $y \in R$

Weak learning algorithm (Weak Learner)

The number of iterations (machines): $\mathrm{T}$ ( $\mathrm{T}$ is an integer) Threshold $\phi(0<\phi<1)$ for demarcating correct and incorrect predictions

2) Initialize:

Number of machine or iteration $t=1$

Distribution $D_{t}(i)=1 / m$ for all $i$

Error rate $\varepsilon_{t}=0$

3) Iterate while $t \leq T$ :

Call Weak Learner, providing it with distribution $D_{t}$

Build the regression model: $f_{t}(x) \rightarrow y$

Calculate absolute relative error for each training example as

$$
\operatorname{ARE}_{t}(i)=\left|\frac{f_{t}\left(x_{i}\right)-y_{i}}{y_{i}}\right|
$$

Calculate the error rate of $f_{t}(x): \varepsilon_{t}=\sum_{i: A R E_{t}(i)>\phi} D_{t}(i)$ Set $\beta_{t}=\varepsilon_{t}^{n}$, where $n=1,2$ or 3 (linear, square, or cubic) Update distribution $D_{t}$

$$
D_{t+1}(i)=\frac{D_{t}(i)}{Z_{t}} \times\left\{\begin{array}{ll}
\beta_{t} & \text { if } A R E_{t}(i) \leq \phi \\
1 & \text { otherwise }
\end{array}\right\}
$$

Where $Z_{t}$ is a normalization factor chosen such that $D_{t+1}$ will be a distribution

Set $t=t+1$ 


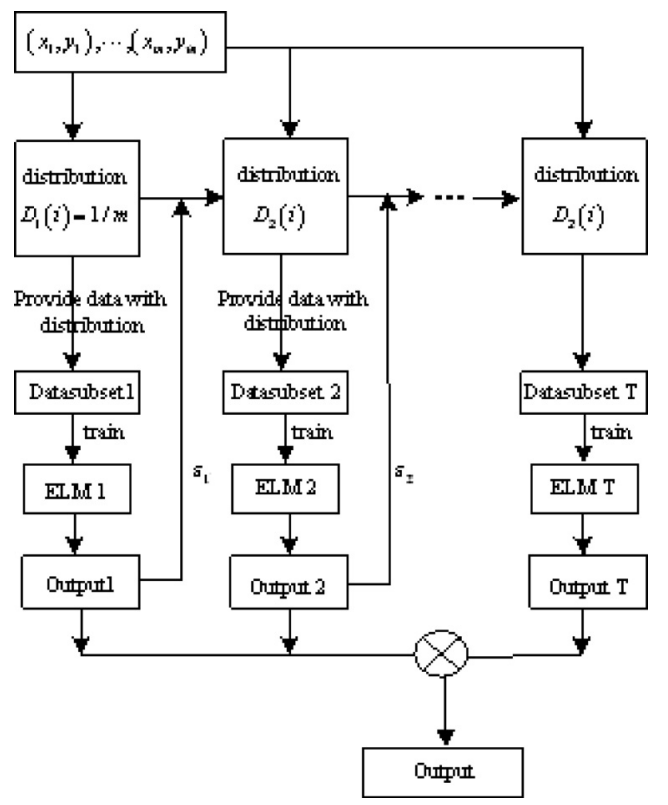

Fig. 2. The ensemble ELM algorithm using the modified AdaBoost.RT algorithm.

Output the final hypothesis:

$$
f_{\text {fin }}(x)=\frac{\sum_{t}\left(\log \frac{1}{\beta}\right) f_{t}(x)}{\sum_{t}\left(\log \frac{1}{\beta}\right)}
$$

\subsection{Modified AdaBoost.RT}

The experiments with the AdaBoost.RT have shown that the performance of the committee machine is sensitive to the value of $\phi .^{11)}$ If $\phi$ is too low, it is generally very difficult to get a sufficient number of correctly predicted examples. Conversely, if $\phi$ is too high, it is not beneficial to get out of the noise and boost the "hard" examples. The above cases affect the performance of the committee machine seriously, and may make the committee machine unstable. In order to estimate $\phi$ efficiently, a self-adaptive method to modify the value of $\phi$ is proposed instead of the invariable value. The value of $\phi$ decreases as the error $\varepsilon_{t}$ in this iteration is bigger than the error $\varepsilon_{t-1}$ in last iteration. When $\varepsilon_{t}$ is smaller than $\varepsilon_{t-1}, \phi$ increases. The value of $\phi$ is adjusted by this way during the whole process of iterations of AdaBoost.RT algorithm. This method can overcome the limitation of original AdaBoost.RT algorithm which is attribute to estimating preliminary threshold $\phi$. The conclusions have been drawn by Solomatine ${ }^{11)}$ : the committee machine is stable while the value of $\phi$ is between 0 and 0.4 , and at the value of around 0.4, the AdaBoost.RT becomes unstable due to overfitting and the boosting of noise. Therefore $0<\phi<0.4$ is defined at the beginning of AdaBoost.RT algorithm. The improved method of self-adaptive modified value of $\phi$ is described: Calculate the root mean square error of output $e$ in every iteration:

$$
e=\sqrt{\frac{1}{N} \sum_{i=1}^{N}\left(\hat{y}_{i}-y_{i}\right)^{2}}
$$

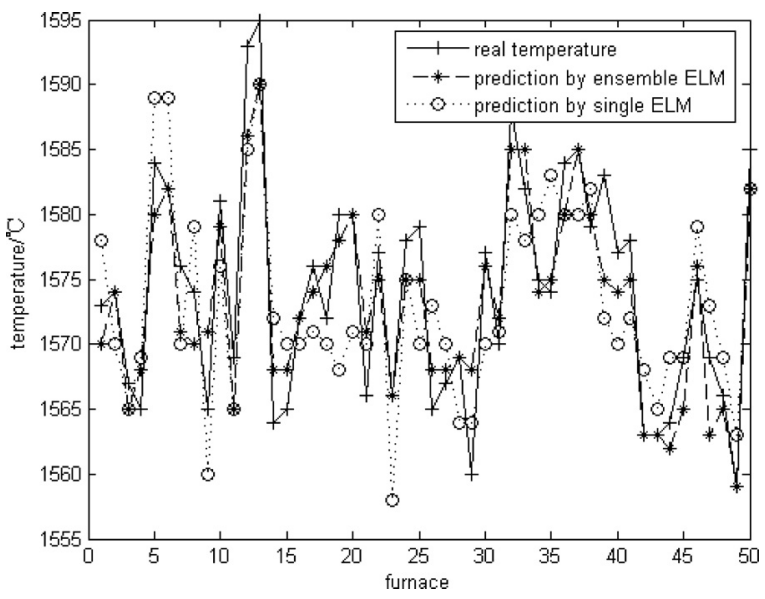

Fig. 3. The comparison of prediction temperatures by hybrid model with ensemble ELM and single ELM "black box" model.

The value of $\phi$ will decrease while $e_{t}<e_{t-1}$, otherwise the value of $\phi$ will increase while $e_{t}>e_{t-1}$. Moreover $\phi_{t+1}=\phi_{t-1}$ while $\left|e_{t}-e_{t-1}\right| \geq d$, where $d$ is a constant. The modification range of $\phi$ is attributed to the change of root mean square error. When the ELM is used as the "weak learner" of the modified AdaBoost.RT, an ensemble ELM intelligent algorithm is obtained. The hybrid intelligent algorithm is shown in Fig. 2.

\section{Experience}

The coefficients of thermal model are predicted by ensemble ELM. The main factors that affect the coefficient $\eta$ and $f_{\text {Surf }}$ are obtained by analyzing the LF metallurgic process. They are the weights of molten steel, temperature of molten steel, the area of molten slag and the refining power consumption. The input layer of ELM has 4 nodes, the hidden layer has 10 nodes and the output layer has 2 nodes ( $\eta$ and $f_{\text {Surf }}$ ). The AdaBoost.RT iterates 10 times. Two hundred and fifty data of production from $300 \mathrm{t}$ LF in Baoshan Iron \& Stee Co. Ltd. from June to October in 2006 are used. They are divided into two groups. The $80 \%$ of them are selected randomly to train the hybrid model, and the others are used to test. For comparing the performance of the hybrid model, a single ELM "black box" method is done to predict the temperature. The input layer of ELM has 6 nodes. They are the refining power consumption, the initial temperature, ladle states, the heat effects of additions, the weight of molten steel and the refining time. The hidden layer has 14 nodes and the output layer has 1 node (temperature). Figure 3 shows the results of predicted temperature by the hybrid model combined the ensemble ELM with mechanism method and the single ELM "black box" model. The results of experience demonstrate that the performance of the single ELM "black box" model is worse than the hybrid model combined the mechanism method with ensemble ELM. In addition, the experience with the hybrid model using traditional classic gradient-based learning algorithm back-propagation neural network (BPNN) as the "weak learner" of modified AdaBoost.RT is done for comparing the performance of hybrid models with different "weak learner". The structure of BPNN is the same as the 


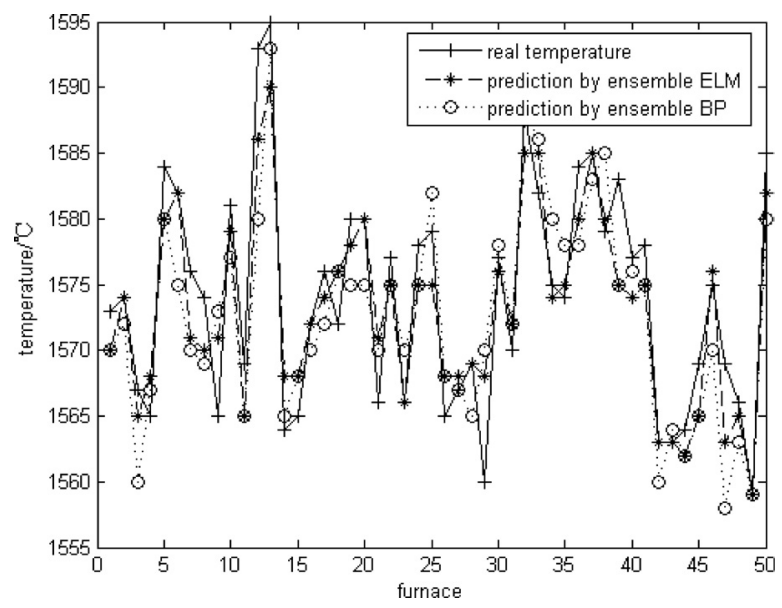

Fig. 4. The comparison of prediction temperatures by hybrid model with ensemble ELM and ensemble BPNN.

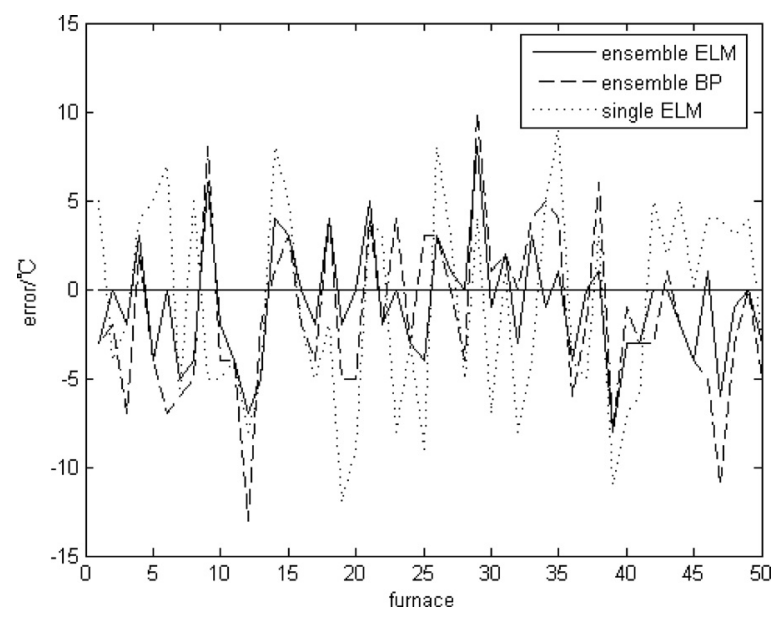

Fig. 5. The comparison of the errors of the hybrid model with ensemble ELM, the hybrid model ensemble BPNN and the "black box" mode model of single ELM.

ELM's. The experience shows that the speed of hybrid model using ELM is faster than the one using BPNN, and the hybrid model using ELM has better accuracy (It is because the ELM may not face the issues like local minima, improper learning rate and overfitting, etc. that the traditional classic gradient-based learning algorithms have to face.). Figure 4 shows the results of predicted temperature by the hybrid model combined mechanism method with the ensemble ELM and with the ensemble BPNN. The prediction errors of three methods are presented in the Fig. 5. It shows the performance of the hybrid model combined the mechanism method with ensemble ELM is better than the others. The new hybrid model has good generalization per- formance and good accuracy. The number of heats of which the predictive errors of temperature of molten steel in LF are not over $5^{\circ} \mathrm{C}$ is greater than $90 \%$. The results indicate that the accuracy of the temperature prediction model is satisfied for metallurgic process.

\section{Conclusion}

In this paper a hybrid method combined intelligent algorithm with mechanism method is presented to predict the molten steel temperature in LF. The thermal model section is based on the analysis of the energy going into and out of the molten steel during the LF refining production process. The ensemble ELM is proposed to predict the coefficients of thermal model which are estimated hardly using traditional mechanism method. The modified AdaBoost.RT is used as the ensemble method. ELM is the "weak learner". It overcomes the limitation of the traditional classic BPNN algorithm. The main factors that affect the coefficients are obtained by analyzing the LF metallurgic process. This hybrid model is used in $300 \mathrm{t} \mathrm{LF}$ of Baoshan Iron \& Stee Co. Ltd. The experiments demonstrate that both the generalization performance and the accuracy of model are improved by combining the mechanism method with intelligent algorithm.

\section{Acknowledgement}

This research is supported by National Natural Science Foundation of China (Grant No. 60674063), Key Laboratory of Process Industry Automation of Northeastern University of Ministry of Education and Liaoning Province

\section{REFERENCES}

1) U. Camdali, M. Tunc and F. Dikec: Appl. Therm. Eng., 21 (2001), 643.

2) U. Camdali and M. Tunc: Can. Metall. Q., 42 (2003), 439.

3) U. Camdali and M. Tunô: J. Iron Steel Res. Int., 13 (2006), 18.

4) Y.-G. Sun, D.-X. Wang, B.-S. Tao, T. Yan, Y. Shi, S.-B. Fang and Y.H. Wang: Proc. of the 3th World Cong. on Intelligent Control and Automation, Hefei, P.R. China, (2000).

5) A. H. Castillejos E., F. A. Acosta G. and A. Betancourt S. et al:: Ironmaking Steelmaking, 6 (1997), 53.

6) O. Vokova and D. Janke: ISIJ Int., 43 (2003), 1185.

7) Subagyo and G. A. Brooks: ISIJ Int., 43 (2003), 1286.

8) G.-B. Huang, Q.-Y. Zhu and C.-K. Siew: Neurocomputing, 70 (2006), 489

9) D. P. Solomatine and D. L. Shrestha: In Proc. of the Int. Joint Conf. on Neural Networks, Piscataway, (2004), 1163.

10) H. Drucker: Proc. of the Fourteenth Int. Conf. on Machine Learning, Morgan-Kaufmann, San Fransisco, CA, (1997), 107.

11) D. L. Shrestha and D. P. Solomatine: Neural Comput., 18 (2006), 1678 . 